\title{
Solitary plane waves in an isotropic hexagonal lattice
}

\author{
Zolotaryuk, Yaroslav; Savin, A.V.; Christiansen, Peter Leth
}

Published in:

Physical Review B Condensed Matter

Link to article, DOI:

10.1103/PhysRevB.57.14213

Publication date:

1998

Document Version

Publisher's PDF, also known as Version of record

Link back to DTU Orbit

Citation (APA):

Zolotaryuk, Y., Savin, A. V., \& Christiansen, P. L. (1998). Solitary plane waves in an isotropic hexagonal lattice. Physical Review B Condensed Matter, 57(22), 14213-14227. https://doi.org/10.1103/PhysRevB.57.14213

\section{General rights}

Copyright and moral rights for the publications made accessible in the public portal are retained by the authors and/or other copyright owners and it is a condition of accessing publications that users recognise and abide by the legal requirements associated with these rights.

- Users may download and print one copy of any publication from the public portal for the purpose of private study or research.

- You may not further distribute the material or use it for any profit-making activity or commercial gain

- You may freely distribute the URL identifying the publication in the public portal

If you believe that this document breaches copyright please contact us providing details, and we will remove access to the work immediately and investigate your claim 


\title{
Solitary plane waves in an isotropic hexagonal lattice
}

\author{
Y. Zolotaryuk \\ Department of Mathematical Modelling, Technical University of Denmark, DK-2800 Lyngby, Denmark \\ and Department of Mathematics, Heriot-Watt University, Edinburg EH14 4AS, United Kingdom \\ A. V. Savin \\ Department of Mathematical Modelling, Technical University of Denmark, DK-2800 Lyngby, Denmark \\ and Institute for Problems of Physics and Technology, 119034 Moscow, Russian Federation \\ P. L. Christiansen \\ Department of Mathematical Modelling, Technical University of Denmark, DK-2800 Lyngby, Denmark
}

(Received 13 January 1998)

Solitary plane-wave solutions in a two-dimensional hexagonal lattice which can propagate in different directions on the plane are found by using the pseudospectral method. The main point of our studies is that the lattice model is isotropic and we show that the sound velocity is the same for different directions of wave propagation. The pseudospectral method allows us to obtain solitary wave solutions with very narrow profile, the thickness of which may contain a few atoms or even less than one lattice spacing (i.e., essentially discrete solutions). Since these nonlinear waves are quite narrow, details of lattice microstructure appear to be important for their motion. Particularly, the regime of their propagation qualitatively depends on whether or not the direction of their motion occurs along the lattice bonds. Two types of solitary plane waves are found and studied. The stability of these solitary waves is investigated numerically by their interactions with vacancies and lattice edges. Propagation of solitary plane waves through finite lattice domains with isotopic disorder is extensively studied. Comparison of these results with the soliton propagation in one-dimensional lattices with mass impurities is presented. [S0163-1829(98)00522-0]

\section{INTRODUCTION}

Solitary waves in two-dimensional (2D) lattices have been studied in a number of publications, ${ }^{1-7}$ using some essential simplifications. Thus, in all of these works scalar models or strongly anisotropic models were considered. Therefore any extension of these studies to isotropic vector models is of interest. The present paper aims to investigate the dynamics of solitary plane waves in the 2D hexagonal lattice shown in Fig. 1. This work is a natural extension of the nonlinear dynamics of one-dimensional (1D) lattices. New features in the dynamics appear when 1D and multidimensional systems are compared. Thus, in a 2D isotropic lattice with microstructure the properties of a nonlinear wave should depend on the direction of propagation.

The 2D hexagonal lattice studied in this paper is an entirely vector model which includes interparticle interactions of the central-symmetric type and only between the nearest neighbors. We consider the realistic $(12,6)$ Lennard-Jones (LJ) potential which contains the core. The presence of this core leads to some specific features of the soliton solution, e.g., the existence of the upper bound for the soliton amplitude. Therefore, it would be interesting to study the soliton dynamics in the 1D LJ lattice as well. We start in this paper with the $1 \mathrm{D}$ case and afterwards pass to the 2D lattice. Because the lattice solitons are essentially discrete objects and the techniques of the continuum analysis are not valid anymore, we find the soliton solutions by applying the pseudospectral method suggested and developed previously by Eilbeck and Flesch ${ }^{8}$ and Duncan et al. ${ }^{9}$ This method allows us to find numerically the traveling-wave solutions for all admissible velocities, the profile of which can be obtained with any given accuracy. In dependence on the direction of solitary wave propagation, we show that in the 2D lattice the solitary plane wave has either a finite or infinite interval of supersonic velocities.

Realistic crystals always contain mass impurities. In order to investigate the stability of the solitary wave propagation in the 2D lattice, we also consider interactions of these waves

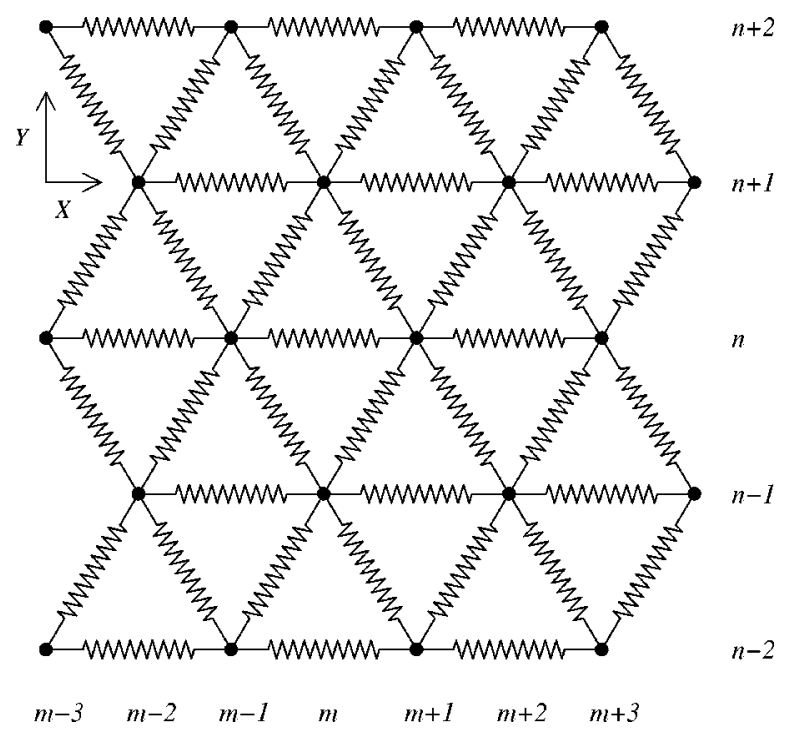

FIG. 1. Schematic representation of the 2D isotropic hexagonal lattice. The nearest-neighbor interactions are shown by springs. 
with mass impurities. In this paper we study the solitary wave propagation through a lattice vacancy (the absence of a particle at some lattice site) and through a 2D domain with isotopic disorder. Also, we will study the influence of different boundary conditions on the solitary wave motion. Contrary to the 1D case, when the lattice soliton is known to be reflected from a heavy mass impurity, the solitary plane wave rounds it, retaining its shape with some radiation of delocalized waves.

The paper is organized as follows. In Sec. II we consider briefly the 1D LJ lattice. The 2D LJ hexagonal lattice model is described in the next section. In Sec. IV we reduce the 2D problem to an effective 1D chain. Soliton dynamics in lattices with mass impurities is studied in Sec. V. Conclusions are given in the next section. Finally, Appendixes A and B contain short descriptions of the pseudospectral method and a soliton solution of the reduced $1 \mathrm{D}$ problem in the continuum approximation.

\section{THE HOMOGENEOUS 1D LJ LATTICE}

We consider a 1D lattice of particles (atoms or molecules) with mass $m$ coupled by the $(12,6) \mathrm{LJ}$ potential. The dimensionless Hamiltonian of such a chain has the form

$$
H=\sum_{n}\left[\frac{1}{2} \dot{u}_{n}^{2}+U\left(u_{n+1}-u_{n}\right)\right],
$$

where the dot denotes the differentiation with respect to the dimensionless time $\tau=\sqrt{m / \kappa} t$ with $\kappa$ being the characteristic stiffness constant of the interatomic LJ potential. The lattice dimensionless variable $u_{n}$ describes the displacement of the $n$th chain particle from its equilibrium position measured in units of the lattice spacing $l$. The dimensionless potential function $U$ is defined through the expression

$$
U(r)=\frac{1}{72}\left[(r+1)^{-6}-1\right]^{2},
$$

so that this function is normalized by the relations $U(0)$ $=0$ and $U^{\prime \prime}(0)=1$. The corresponding equations of motion are

$$
\ddot{r}_{n}=U^{\prime}\left(r_{n+1}\right)-2 U^{\prime}\left(r_{n}\right)+U^{\prime}\left(r_{n-1}\right), \quad n=0, \pm 1, \ldots,
$$

where $r_{n}=u_{n+1}-u_{n}$ is the relative displacement from the equilibria of the $n$th and the $(n+1)$ th lattice particles.

We look for solitary wave solutions of Eq. (3) propagating with constant velocity $s: r_{n}(\tau)=r(n-s \tau)=r(z), z=n$ $-s \tau$. The boundary conditions for these types of solutions are $r( \pm \infty)=0$ and $r^{\prime}( \pm \infty)=0$. These solutions can be found by using the pseudospectral method described in Appendix A. For the description of soliton solutions it is convenient to use such quantities as their amplitude $A=-r(0)$, the "thickness" (diameter)

$$
D=2\left[\int_{0}^{\infty} z^{2} r(z) d z / \int_{0}^{\infty} r(z) d z\right]^{2},
$$

and the energy defined according to the Hamiltonian (1). The soliton energy $E$ can be given in terms of the functions $r(z)$ and $r^{\prime}(z)$ obtained by using the pseudospectral method:

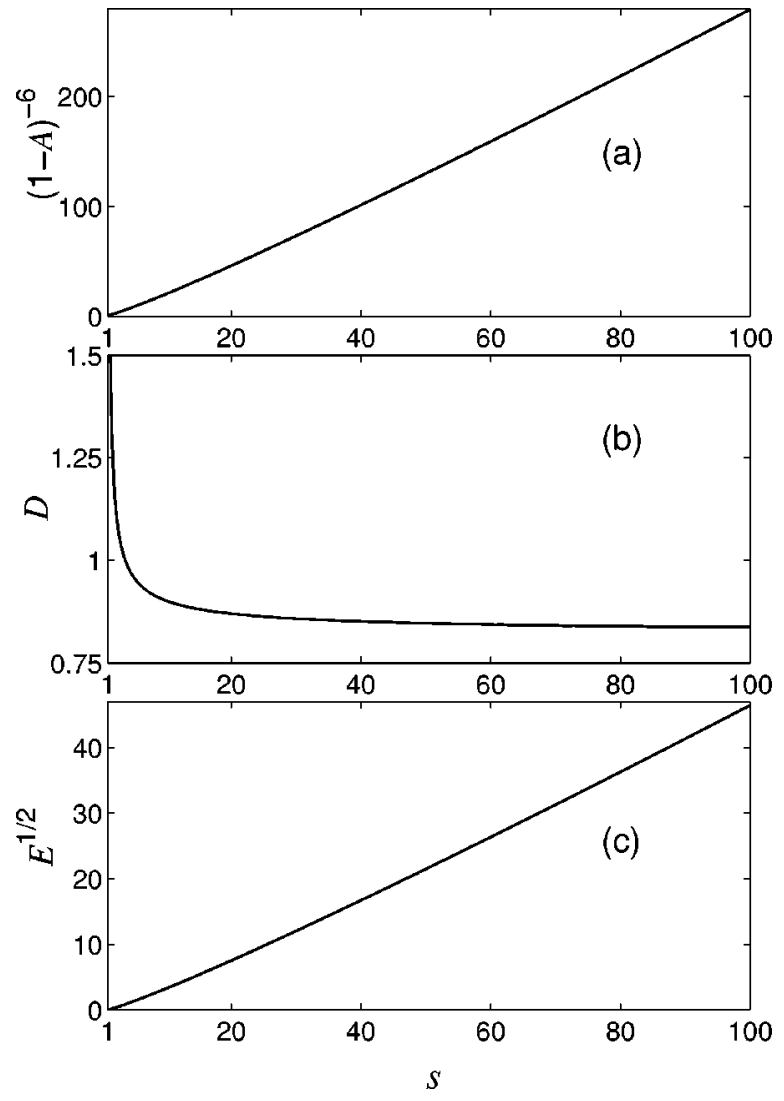

FIG. 2. (a) Function $(1-A)^{-6}$ of the soliton amplitude $A$, (b) soliton diameter $D$, and (c) square root of the soliton energy $\sqrt{E}$ against the soliton velocity $s$ in the 1D LJ lattice.

$$
E=\sum_{n=-N_{D}}^{N_{D}}\left[\frac{s^{2}}{2}\left(\sum_{j=-N_{D}}^{n} r_{j}^{\prime}\right)^{2}+U\left(r_{n}\right)\right],
$$

where $r_{n}=r(n)$ and $r_{n}^{\prime}=r^{\prime}(n)$ with the boundary condition $u_{-N_{D}}^{\prime}=0$. The number $N_{D}$ chosen to be, for instance, the integer part of the number $10 D$ provides that the soliton is situated far away from the boundaries.

The numerical investigation of Eqs. (3) for the LJ chain shows that the lattice soliton solutions exist for all supersonic $(s>1)$ velocities. The behavior of the soliton amplitude $A$ as a function of the velocity $s$ is shown in Fig. 2(a) where the function $(1-A)^{-6}$ against $s$ is plotted. This means that the amplitude $A$ tends monotonically to 1 as $1-O\left(s^{-1 / 6}\right)$. The soliton thickness $D$ decreases monotonically with saturation as shown in Fig. 2(b). When $s=1.0055$, the width is less than 10 , when $s=1.0226$, becomes less than 5 , next for $s$ $=1.175$ it becomes less than 2 , and finally for $s=3.7$ it becomes even less than one lattice spacing, i.e., 1 . The soliton energy $E$ increases monotonically as $s^{2}$ [see Fig. 2(c)].

The LJ lattice is not an integrable system. Let us consider the head-in collision of two solitons. During the collision the soliton interaction causes the radiation of small-amplitude waves (phonons). The nonelasticity of this collision can be described by the energy-loss percentage defined by $p$ $=\left[\left(E_{1}-E_{2}\right) / E_{1}\right] 100 \%$ where $E_{1}$ is the soliton energy before collision and $E_{2}$ the soliton energy after collision. The dependence of the energy-loss percentage $p$ on the soliton velocity $s$ is presented in Table I. As follows from this table, 
TABLE I. Dependence of energy losses $p$ after head-in collision of two solitons in the 1D LJ lattice on their velocity $s$.

\begin{tabular}{lcccccccccc}
\hline \hline$s$ & 1.03 & 1.06 & 1.1 & 1.2 & 1.23 & 1.3 & 2.0 & 2.5 & 3.0 & 3.5 \\
\hline$p(\%)$ & $1.4 \times 10^{-5}$ & $1.1 \times 10^{-4}$ & $3.7 \times 10^{-4}$ & $8.1 \times 10^{-4}$ & $8.3 \times 10^{-4}$ & $7.6 \times 10^{-4}$ & $5.6 \times 10^{-5}$ & $7.9 \times 10^{-6}$ & $9.8 \times 10^{-7}$ & $4.6 \times 10^{-8}$ \\
\hline \hline
\end{tabular}

the maximal energy loss occurs at the velocity $s=1.23$ and equals 0.000 81. The collision process is illustrated by Fig. 3(a). The phonon radiation is visible only after significant zooming as shown in Figs. 3(b)-3(d). The small values of $p$ mean that the LJ chain is close to be a completely integrable system. Energy losses tend to zero when $s \rightarrow 1+0$ [in this limit the soliton dynamics is described by the completely integrable Korteweg-de Vries equation) and when $s \rightarrow \infty$ (in this limit soliton dynamics is described by the completely integrable rigid-sphere model).

\section{2D LJ LATTICE}

Let us consider a 2D isotropic hexagonal lattice. Its sites are labeled as shown in Fig. 1. Each lattice particle interacts with its six nearest neighbors which are situated at the dis- tance 1 from it. The interparticle bonds shown in Fig. 1 by springs are described by the LJ potential (2). We use the Cartesian frame of reference $X Y$, so that each lattice site corresponds to the pair of integers $(m, n)$ with $m$ and $n$ being both either even or odd. We denote this set of integer pairs $(m, n)$ by $\Gamma$. In the equilibrium position the $(m, n)$ th particle has the coordinates $(m / 2, \sqrt{3} n / 2)$. Let $\left(u_{m n}, v_{m n}\right)$ be the $2 \mathrm{D}$ vector displacement of the $(m, n)$ th particle from its equilibrium position. Then the Hamiltonian of the lattice can be written as

$$
H=\sum_{(m, n) \in \Gamma}\left[\frac{1}{2} \mu_{m n}\left(\dot{u}_{m n}^{2}+\dot{v}_{m n}^{2}\right)+\sum_{(k, l)} U\left(r_{k l m n}\right)\right]
$$

where

$$
r_{k l m n}=\sqrt{\left(\frac{k}{2}+u_{m+k, n+l}-u_{m n}\right)^{2}+\left(\frac{\sqrt{3}}{2} l+v_{m+k, n+l}-v_{m n}\right)^{2}}-1
$$

is the deviation from the equilibrium distance between the $(m+k, n+l)$ th and the $(m, n)$ th particles and the summation over $(k, l)$ runs three bonds in each lattice cell. We choose the following three pairs: $(2,0),(1,1)$, and $(-1,1)$. The corresponding equations of motion can be written in the standard manner from the Hamiltonian (6).

\section{SOLITARY PLANE WAVES}

Let us consider the motion of a plane wave in a general direction given by the Miller indices $[\bar{i} j 0]$ which are defined in the frame of reference formed by the basis vectors, the direction of which coincides with adjacent bonds. The direction of wave propagation coincides with the line that connects the origin $(0,0)$ with the particle at the lattice site $(m, n)=(2 i+j, j)$. The angle between this line and the $X$ axis is $\alpha=\tan ^{-1}(\sqrt{3} n / m)=\tan ^{-1}[\sqrt{3} j /(2 i+j)]$. Without loss of generality (because of the hexagonal symmetry), we can assume that $0 \leqslant n \leqslant m / 3, m>1,(0 \leqslant \alpha \leqslant \pi / 6)$. Now we rotate the Cartesian frame $X Y$ by the angle $\alpha$ anticlockwise. Then in the new frame $(u, v)$ the $u$ axis determines the direction of wave propagation and the $v$ axis coincides with the plane (front) of a wave (note that in the $2 \mathrm{D}$ case the "plane (front) of a wave", is just a line).

We define a "directed elementary cell" of the 2D hexagonal lattice which corresponds to the angle of wave propagation $\alpha$ as the smallest rectangular formed by the vertices at the lattice sites $(0,0),(m, n),\left(m+m_{1}, n+n_{1}\right)$, and $\left(m_{1}, n_{1}\right)$ where $m_{1}=-n$ and $n_{1}=m / 3$ if $m$ is proportional to 3 and $m_{1}=-3 n, n_{1}=m$ if $m$ is not proportional to 3 (see Fig. 4). The length of the rectangular sides which are parallel to the $u$ and $v$ axes are given by the expressions

$$
l_{u}=\frac{1}{2} \sqrt{m^{2}+3 n^{2}}, \quad l_{v}=\frac{1}{2} \sqrt{m_{1}^{2}+3 n_{1}^{2}},
$$

respectively. When the plane-wave propagates in the direction of the $u$ axis, all the lattice sites which are situated along a line, parallel to the wave front, are equally displaced, so that only the $u$ projections of the sites change. Therefore the problem of the plane-wave propagation in the $2 \mathrm{D}$ lattice can be reduced to the effective $1 \mathrm{D}$ model.

Both as in the 2D lattice and in the "reduced" 1D chain, each particle interacts with its six (not necessarily nearest) neighbors. The length of the undistorted bonds in the reduced chain corresponds to the length of the projections of the three bonds outcoming from the site $(0,0)$ on the $u$ axis:

$$
\begin{gathered}
d_{1}=\cos \left(\frac{\pi}{3}+\alpha\right)=\frac{m-3 n}{2 \sqrt{m^{2}+3 n^{2}}} \geqslant 0, \\
d_{2}=\cos \left(\frac{\pi}{3}-\alpha\right)=\frac{m+3 n}{2 \sqrt{m^{2}+3 n^{2}}}>0, \\
d_{3}=\cos \alpha=\frac{m}{\sqrt{m^{2}+3 n^{2}}}>0 .
\end{gathered}
$$



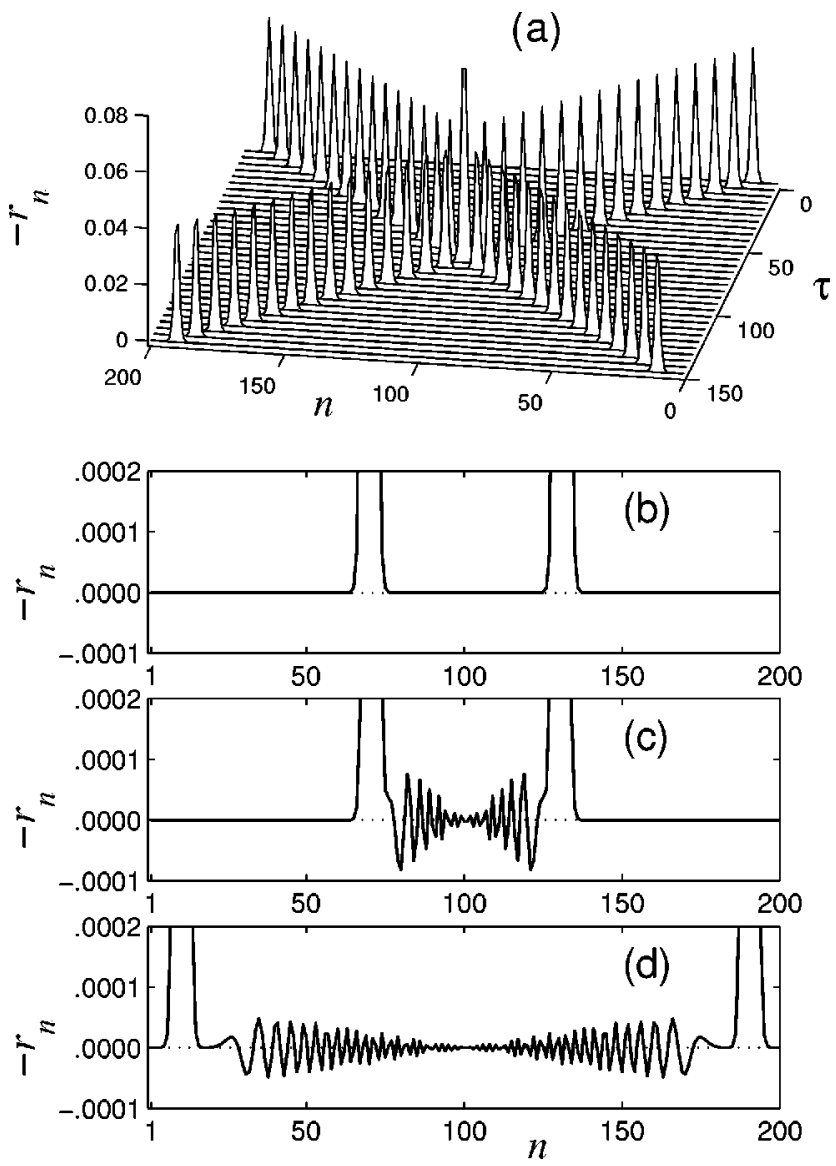

FIG. 3. (a) Head-in collision of the lattice solitons with velocities $s=1.2$ in the 1D LJ lattice, (b) their profiles before collision ( $\tau=50)$, (c) after collision ( $\tau=100)$, and (d) later, at $\tau=150$.

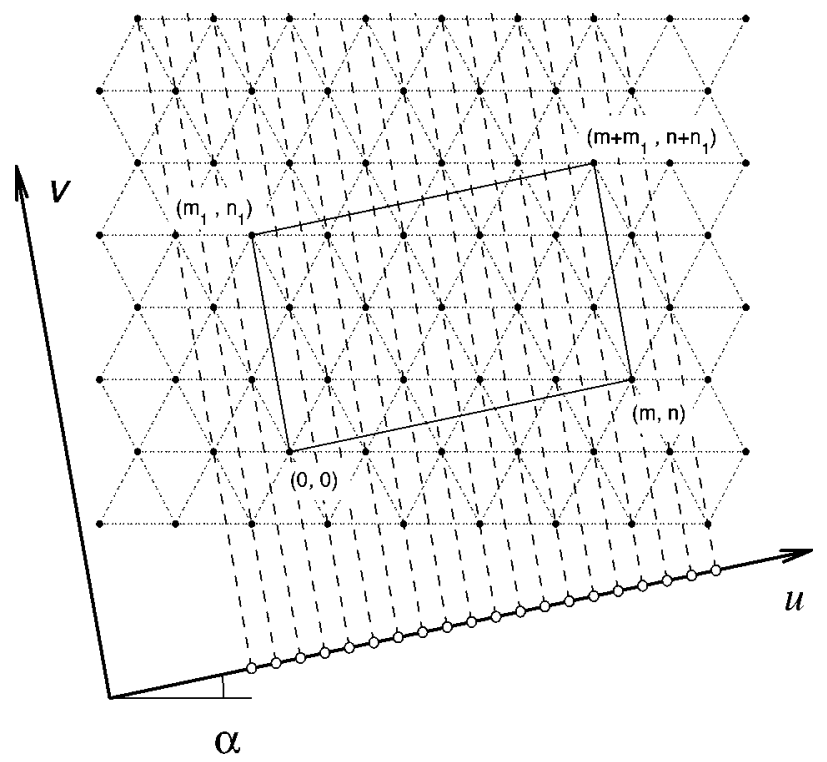

FIG. 4. Construction of the reduced 1D chain model corresponding to motion of a solitary plane wave in the direction given by $\alpha$. The particular case of the directed elementary cell with $m$ $=9$ and $n=1\left(\alpha=10.89^{\circ}\right)$ is presented. For this case the vertices of the cell are $(0,0),(9,1),(8,4)$, and $(-1,3)$. Its boundaries are indicated by solid lines of length $l_{u}$ and $l_{v}$ defined by Eqs. (8). Projections of lattice sites on the $u$ axis are shown by dashed lines.

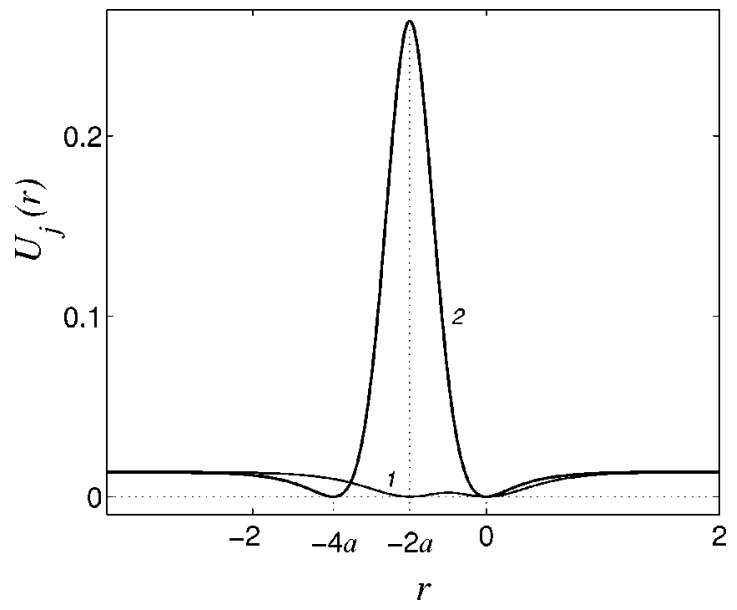

FIG. 5. Shape of the reduced LJ potential $U_{j}(r)$ for $j=1$ (curve 1) and $j=2$ (curve 2). Spacing of the reduced 1D chain is $a$ $=0.327\left(m=9, n=1\right.$, and $\left.\alpha=10.89^{\circ}\right)$.

Since $(m, n) \in \Gamma$, the numbers $(m-3 n) / 2,(m+3 n) / 2$, and $m$ are integers. Let $l \geqslant 1$ be their largest common divisor, i.e., $(m-3 n) / 2=k_{1} l,(m+3 n) / 2=k_{2} l$, and $m=k_{3} l$, where $k_{1}$, $k_{2}$, and $k_{3}$ are integers. Therefore the spacing of the reduced chain can be defined by

$$
a=a(\alpha)=\frac{d_{j}}{k_{j}}=\frac{l}{\sqrt{m^{2}+3 n^{2}}}, \quad j=1,2,3 .
$$

The Hamiltonian of this chain can be written in the form

$$
H=\frac{1}{l_{v}} \sum_{n}\left[\frac{1}{2} \dot{u}_{n}^{2}+\sum_{j=1}^{3} U_{j}\left(u_{n+k_{j}}-u_{n}\right)\right],
$$

where $u_{n}$ is the displacement of the $n$th particle in this chain and the $j$ th $(j=1,2,3)$ reduced LJ potential is defined by

$$
U_{j}(r)=\frac{1}{72}\left[\left(1+2 d_{j} r+r^{2}\right)^{-3}-1\right]^{2}, \quad d_{j}=k_{j} a
$$

if $k_{j} \neq 0$ and $U_{j}(r) \equiv 0$ if $k_{j}=0, j=1,2,3$. The Hamiltonian (11) gives the energy density along the wave front. The reduced LJ potential (12) coincides with the standard LJ potential only if $d_{j}=1$. For other values of $d_{j}$ the reduced potential (12) is a function bounded from above as shown in Fig. 5. Its maximal values are given by

$$
U_{j, \max }=\max \left\{\frac{1}{72}\left[\left(1-d_{j}^{2}\right)^{-3}-1\right]^{2}, \frac{1}{72}\right\},
$$

at $r=-d_{j}$ while its minima $\left(U_{j, \min }=0\right)$ happen at $r=$ $-2 d_{j}$ and 0 (see Fig. 5).

The equations of motion that correspond to the Hamiltonian (11) are

$$
\begin{gathered}
\ddot{u}_{n}=\sum_{j}\left[U_{j}^{\prime}\left(u_{n+k_{j}}-u_{n}\right)-U_{j}^{\prime}\left(u_{n}-u_{n-k_{j}}\right)\right] \\
n=0, \pm 1, \ldots
\end{gathered}
$$




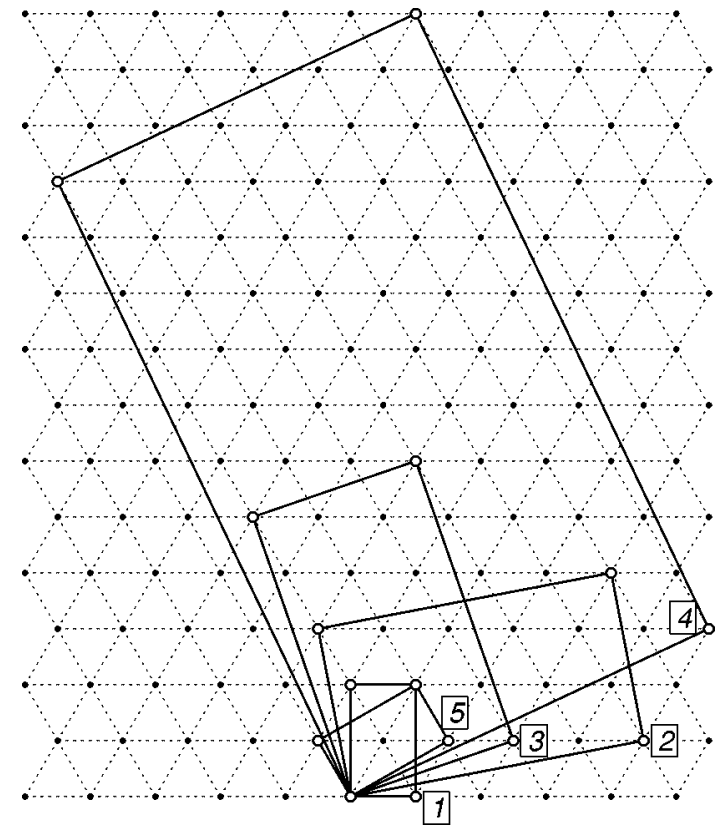

FIG. 6. Five directed elementary cells (shown by the rectangulars) for the five directions of solitary wave propagation. The rectangulars 1, 2, 3, 4, and 5 correspond to the directions [010] ( $\alpha$ $\left.=0^{\circ}\right), \quad[\overline{4} 10] \quad\left(\alpha=10.89^{\circ}\right), \quad[\overline{2} 10] \quad\left(\alpha=19.11^{\circ}\right), \quad[\overline{4} 30] \quad(\alpha$ $\left.=25.29^{\circ}\right)$, and $[\overline{1} 10]\left(\alpha=30^{\circ}\right)$.

Here and throughout this paper the summation over $j$ runs the values: $j=1,2$, and 3 . This system of equations can be rewritten in terms of the longitudinal relative displacements $r_{n}=u_{n+1}-u_{n}$ as follows:

$$
\begin{aligned}
\ddot{r}_{n}= & \sum_{j}\left[U_{j}^{\prime}\left(\sum_{l=1}^{k_{j}} r_{n+l}\right)-U_{j}^{\prime}\left(\sum_{l=1}^{k_{j}} r_{n+l-1}\right)\right. \\
& \left.-U_{j}^{\prime}\left(\sum_{l=1}^{k_{j}} r_{n-k_{j}+l}\right)+U_{j}^{\prime}\left(\sum_{l=1}^{k_{j}} r_{n-k_{j}-1+l}\right)\right] .
\end{aligned}
$$

In the continuum limit the system of the discrete Eqs. (15) can be transformed to the Boussinesq equation and its solution is given in Appendix B. The soliton solution exists for all $s>s_{0}$ where $s_{0}=\sqrt{9 / 8}$ is the velocity of small-amplitude waves in the lattice which does not depend on the direction of wave propagation.

Similarly to the 1D case, in order to find numerically exact soliton solutions which take into account the discreteness of the lattice:

TABLE II. Geometric parameters $[\bar{i} j 0],(m, n),\left(m_{1}, n_{1}\right), \alpha$; $l_{u}, l_{v} ; k_{1}, k_{2}, k_{3} ; N_{\alpha}, a$ for five directions of solitary wave propagation.

\begin{tabular}{lcccccccccc}
\hline \hline$[\bar{i} j 0]$ & $(m, n)$ & $\left(m_{1}, n_{1}\right)$ & $\alpha$ & $l_{u}$ & $l_{v}$ & $k_{1}$ & $k_{2}$ & $k_{3}$ & $N_{\alpha}$ & $a$ \\
\hline$[010]$ & $(2,0)$ & $(0,2)$ & $0^{\circ}$ & 1 & $\sqrt{3}$ & 1 & 1 & 2 & 2 & 0.5 \\
{$[\overline{4} 10]$} & $(9,1)$ & $(-1,3)$ & $10.89^{\circ}$ & $\sqrt{21}$ & $\sqrt{7}$ & 1 & 2 & 3 & 14 & 0.327 \\
{$[\overline{2} 10]$} & $(5,1)$ & $(-3,5)$ & $19.11^{\circ}$ & $\sqrt{7}$ & $\sqrt{21}$ & 1 & 4 & 5 & 14 & 0.189 \\
{$[\overline{4} 30]$} & $(11,3)$ & $(-9,11)$ & $25.29^{\circ}$ & $\sqrt{37}$ & $\sqrt{111}$ & 1 & 10 & 11 & 74 & 0.082 \\
{$[\overline{1} 10]$} & $(3,1)$ & $(-1,1)$ & $30^{\circ}$ & $\sqrt{3}$ & 1 & 0 & 1 & 1 & 2 & 0.866 \\
\hline \hline
\end{tabular}
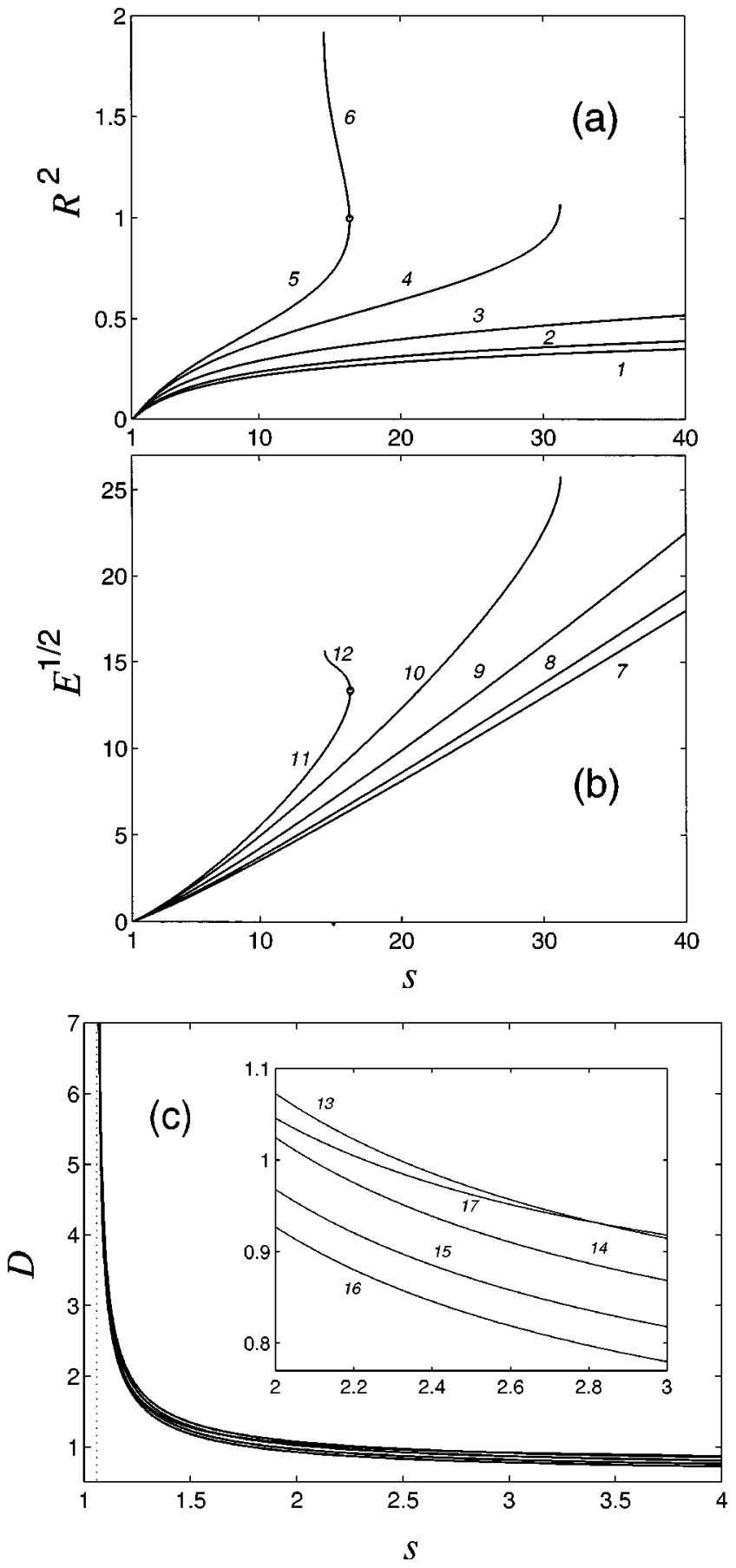

FIG. 7. (a) Squared total lattice compression $R^{2}$, (b) square root $\sqrt{E}$ of the energy $E$ given by the Hamiltonian (11), and the profile thickness $D$ against velocity $s$ for the solitary wave propagating in the directions $\alpha=0^{\circ}$ (curves 1, 7, and 13), $\alpha=10.89^{\circ}$ (curves 2, 8, and 14), $\alpha=19.11^{\circ}$ (curves 3, 9, and 15), $\alpha=25.29^{\circ}$ (curves 4, 10, and 16), and $\alpha=30^{\circ}$ (curves 5, 11, and 17). Two small circles in (a) and (b) are the bifurcation points which separate the hard solitary wave solution represented by curves 5 and 11 from the soft solitary wave solution represented by curves 6 and 12 for the case with $\alpha$ $=30^{\circ}$.

$$
r_{n}(\tau)=r(z), \quad z=n a-s \tau,
$$

we use the pseudospectral method (for details see again Appendix A). For the description of solitary wave solutions we use the total compression of the lattice $R=-\Sigma_{n} r_{n}=u_{-\infty}$ 
TABLE III. Dependence of total lattice compression $R$, profile thickness $D$, and energy $E$ on the direction angle $\alpha$ (velocity is $s$ $=1.5)$.

\begin{tabular}{lccc}
\hline \hline$\alpha$ & $R$ & $D$ & $E$ \\
\hline $0^{\circ}$ & 0.1599 & 1.3458 & 0.02611 \\
$10.89^{\circ}$ & 0.1664 & 1.2934 & 0.02940 \\
$19.11^{\circ}$ & 0.1777 & 1.2275 & 0.03463 \\
$25.29^{\circ}$ & 0.1854 & 1.1817 & 0.03872 \\
$30^{\circ}$ & 0.1886 & 1.2765 & 0.04075 \\
\hline \hline
\end{tabular}

$-u_{\infty}$ calculated in the direction of wave propagation, the thickness (diameter) $D$ of solitary wave profile given by Eq. (4), and the energy $E$ calculated according to Eq. (5) and normalized by $l_{v}$ as in the Hamiltonian (11).

We consider the five directions of wave propagation given

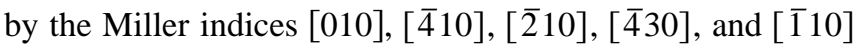
(shown in Fig. 6). The values of the parameters $\left(m_{1}, n_{1}\right), \alpha$, $l_{u}, l_{v}, k_{1}, k_{2}, k_{3}$, and the number $N_{\alpha}$ of sites in the directed elementary cell are presented in Table II. For these five directions of propagation, $R^{2}, \sqrt{E}$, and $D$ against the velocity $s$ are plotted in Fig. 7. The solitary wave has the infinite interval of velocities, $s_{0}<s<\infty$, only in the direction $\alpha=0$ ( $m$ $=2, n=0)$. However, for $0<\alpha \leqslant \pi / 6$ the solitary wave has the finite interval of velocities: $s_{0}<s \leqslant s_{1}<\infty$ where $s_{1}$ $=s_{1}(\alpha)$ is some critical (maximal) value. The finiteness of this interval is a consequence of the boundedness of the reduced LJ potential (12) from above (see Fig. 5). Indeed, as we have shown in Sec. II, the soliton amplitude in the 1D LJ chain is bounded for all velocities $1<s<\infty$ because the particles in such a chain cannot pass each through other. However, in the 2D lattice when $\alpha \neq 0$, the projections of the particle displacements on the $u$ axis can "pass", each through other and this is in agreement with the finite behavior of the reduced potential (12), plotted in Fig. 5, at $r=$ $-d_{j}$. With the growth of the velocity $s$ of the solitary wave solution (16) the amplitude $A=-r(0)$ increases as in the 1D case but this increase happens to be more and more strong while approaching a certain critical value $s_{1}$ because the potential (12) becomes more and more soft. At the point of inflection (where the second derivative of a function equals zero) of this potential which is closest to the point $r=0$, the derivative of the amplitude $A(s)$ with respect to the velocity $s$ becomes infinity, so that for velocities $s>s_{1}$ solitary wave solutions are impossible. In the point of inflexion, hardness of the reduced potential (12) changes into softness which prevents the solitary wave regime. However, since the reduced potential (12) contains also the hard region, we may expect that the solitary wave solutions with amplitude exceeding the critical value $A_{1} \equiv A\left(s_{1}\right)$ can also exist. Indeed, below we confirm that there also exists another branch of solitary wave solutions with an amplitude $A$ in the softness region of the potential (12), so that the critical value $s=s_{1}$ becomes a bifurcation point.

When $\alpha \rightarrow 0+$, the critical (maximal) velocity $s_{1}$ tends to infinity. The increase of $\alpha$ leads to decreasing the upper bound $s_{1}$. Thus, for $\alpha=25.29^{\circ}$ we have $s_{1}=31.23$, while for $\alpha=30^{\circ}$ this bound is $s_{1}=16.42$. For other values of $\alpha$ the critical value $s_{1}$ exceeds 100. As one can see from Figs. 7(a)

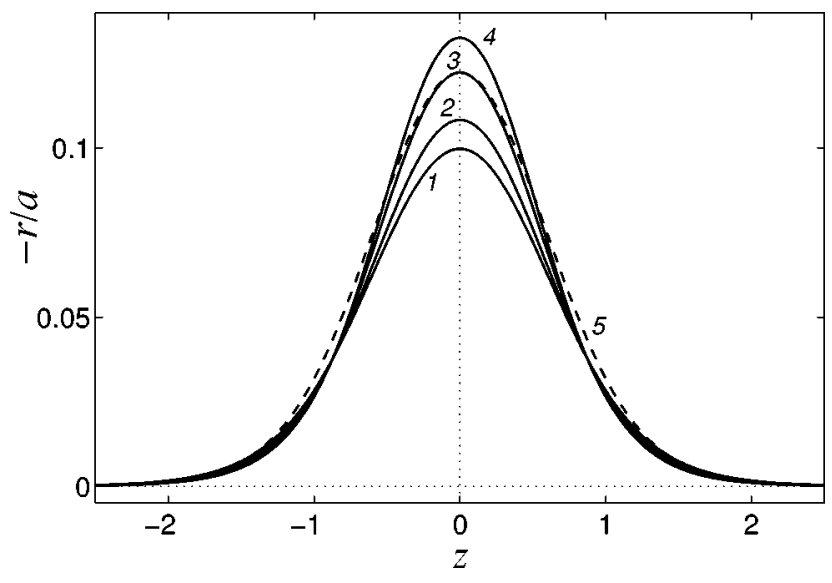

FIG. 8. Normalized profile of the solitary wave $-r(z) / a$ moving in directions with $\alpha=0^{\circ}$ (curve 1), $\alpha=10.89^{\circ}$ (curve 2), $\alpha$ $=19.11^{\circ}$ (curve 3), $\alpha=25.29^{\circ}$ (curve 4), and $\alpha=30^{\circ}$ (curve 5).

and 7(b), the total lattice compression $R$ and the energy $E$ increase with the growth of the angle $\alpha$ within the interval $0^{\circ} \leqslant \alpha \leqslant 30^{\circ}$. The increase of $s$ also results in decreasing the soliton thickness $D$ as demonstrated by Fig. 7(c). As can be seen from this figure, the diameter $D$ depends weakly on the angle of propagation $\alpha$. Exact values of the compression $R$, the diameter $D$, and the energy $E$ for different directions of the solitary wave propagation are presented in Table III. The plots of the corresponding soliton profiles normalized by the spacing $a$ are shown in Fig. 8. As follows from this figure, the change of the propagation direction affects mainly the solitary wave amplitude $A$ but not its shape.

The amplitude $A$ of the solitary wave solutions presented in Fig. 8 by curves $1-5$ lies in the hardness region of the reduced potential (12) and we call these types of solutions the "hard" solitary waves. As shown in Fig. 8, curve 5, which corresponds to the direction of propagation with $\alpha$ $=\pi / 6$, bifurcates at the critical (maximal) velocity $s_{1}$ $=16.42$, into the solitary wave solutions for which its amplitude lies in the softness region of the potential (12). We call these types of solutions the "soft"' solitary waves. The bifurcation of the hard solitary wave solution into the soft solution is illustrated by curves 5 and 6 in Fig. 7(a) and by curves 11 and 12 in Fig. 7(b). The soft solitary wave solution plotted in Fig. 9 by curve 2 has larger amplitude and thickness if compared to the hard solution shown in this figure by

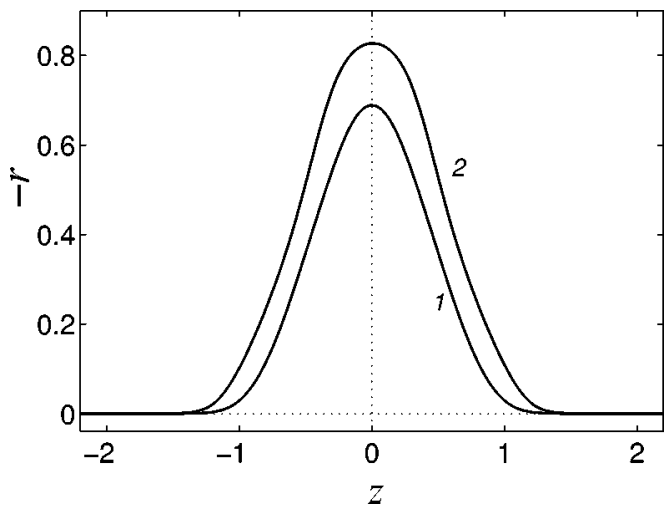

FIG. 9. Profile of the two solitary wave solutions $-r(z)$ propagating with velocity $s=15$ in the direction with $\alpha=30^{\circ}$. 
curve 1. Note that for $\alpha=\pi / 6$, according to Eq. (12), $U_{1}(r) \equiv 0$ and the potentials $U_{2}(r)=U_{3}(r)$ have the point of inflexion at $r=-0.728$. This value appears to be between the amplitudes (the maxima of curves 1 and 2 in Fig. 9) of the hard and soft solitary waves. In this case the soft solution exists for velocities from the interval $14.62 \leqslant s \leqslant s_{1}=16.42$. The peculiar property of this solution is the decrease of its amplitude and energy with the growth of the velocity $s$ (see Fig. 7, curves 6 and 12). The numerical integration of the equations of motion for the reduced chain (15) with the initial conditions obtained by using the pseudospectral method has shown that both types of solitary waves are stable. They propagate in the lattice with constant velocity, retaining their shape. For a soft solitary wave the particles in the vicinity of the wave amplitude are subjected to the soft part of the reduced potential (12) whereas the particles at the tails of the solitary wave profile are affected by the hard part of this potential. Therefore for the existence of soft solitary waves the effect of the hard part of the potential (12) must dominate the effect of the soft part. As a result, their amplitude has to be bounded from above.

\section{INTERACTION OF SOLITARY PLANE WAVES WITH VACANCIES AND MASS IMPURITIES}

As was shown in the previous section, the properties of the propagation of a narrow solitary wave essentially depend on the direction (given by the angle $\alpha$ ) of this propagation. The biggest difference exists at the angle values $\alpha=0$ and $\alpha=\pi / 6$ (or the same, $\alpha=\pi / 2$ ). Therefore we restrict ourselves by investigation only at these two angles.

Let us consider the motion of a solitary wave along the $X$ axis $(\alpha=0)$. In order to simulate properly the solitary wave motion in the infinite 2D lattice, we consider a sufficiently large finite rectangular domain $\Lambda$ contained in $\Gamma$ with 1 $\leqslant m \leqslant M$ and $1 \leqslant n \leqslant N$, and assume at the lattice edges which are perpendicular to the wave propagation, the periodic boundary conditions (i.e., substitute $n+1$ by 1 if $n$ $=N$ and $n-1$ by $N$ if $n=1$ ), whereas the lattice boundaries in the longitudinal direction are assumed to be fixed (i.e., $\dot{u}_{1 n} \equiv 0$ and $\left.\dot{u}_{M n} \equiv 0\right)$. Let the center of a solitary wave be initially $(\tau=0)$ situated (along the $X$ axis) at some site with $m_{0}<M / 2$. Then for the domain $\Lambda$ we can use the following initial conditions:

$$
\begin{gathered}
u_{m n}(0)=\sum_{l=1}^{m} r\left[a\left(l-m_{0}\right)\right], \quad v_{m n}(0)=0, \\
\dot{u}_{m n}(0)=-s \sum_{l=1}^{m} r^{\prime}\left[a\left(l-m_{0}\right)\right], \quad \dot{v}_{m n}(0)=0,
\end{gathered}
$$

where the function $r(z)$ with $s>s_{0}$ is a soliton solution of the reduced system of Eqs. (15) and the period of the reduced chain is $a=1 / 2$ (note that $\alpha=0$ ). After the solitary wave has passed in the longitudinal direction $M / 2$ lattice sites, we shift it back by $M / 2$ sites, modeling in this way the solitary wave motion in the infinite lattice. Therefore, we are able to avoid simulations of lattice dynamics with large numbers of particles.
For modeling the solitary wave motion along the bonds (i.e., when $\alpha=0$ ) we used the number of lattice sites given by $M=N=200$. The solitary wave velocity was taken $s$ $=1.5$. Initially, the solitary wave was placed at $m_{0}=50$ [see Fig. 10(a)]. In order to model the presence of a mass vacancy at the $\left(m_{i}, n_{i}\right)$ th site, it is sufficient in the system of equations of motion governed by the Hamiltonian (6) to set $\mu_{m_{i} n_{i}} \equiv 0$ and to remove all the terms that are responsible for the interparticle interactions with the $\left(m_{i}, n_{i}\right)$ th site. We choose this position to be $m_{i}=n_{i}=100$, so that the vacancy is placed at the center of the lattice rectangular domain. The numerical integration of the equations of motion has shown that the propagation through the vacancy does not destroy the solitary wave [see Fig. 10(b)]. In the vicinity of the vacancy a transverse modulation of the solitary wave appears that creates two transverse waves propagating along the front in opposite directions, as shown in Fig. 10(c). The motion of these waves is accompanied by radiation of longitudinal small-amplitude waves. Due to the periodic boundary conditions, the transverse modulational waves pass through each other many times eventually disappearing, so that the solitary wave becomes undistorted in the limit $\tau \rightarrow \infty$. Before the passage through the vacancy, the solitary wave had the energy $E=4.52$, while after the passage at the time instant $\tau$ $=1567$ the energy was $E=4.48$. Thus, the solitary wave of width $100 \sqrt{3}$ lost at one vacancy only $0.84 \%$ of its energy.

Let us consider now the solitary wave propagation through a lattice domain $\Lambda_{i} \subset \Lambda$ with isotopic disorder. For simulations it was chosen with $40 \leqslant m \leqslant 140$ and $1 \leqslant n$ $\leqslant 200$. We chose at each $n$ a random site number $m(n)$ from the interval $40 \leqslant m \leqslant 140$. Next, we set $\mu_{m(n), n}=\mu_{i}$, where $\mu_{i} \geqslant 1$ is the isotope mass at the $[m(n), n]$ th site and $\mu_{m n}$ $=1$ for the rest of the lattice sites. Then the density of mass impurities in the domain $\Lambda_{i}$ is $c=1 / 50 \sqrt{3}$. The solitary wave propagation through the domain $\Lambda_{i}$ with $\mu_{i}=3$ is shown in Fig. 11. Initially, the solitary wave center was situated at the site $m_{0}=18$ [see Fig. 11(a)]. While passing the domain $\Lambda_{i}$, radiation of small-amplitude waves occurs at each impurity as shown by Fig. 12(b). The solitary wave outcomes from this domain are strongly distorted [see Fig. 12(c)]. The straight linearity of the solitary wave profile is a necessary condition for its motion. The alignment of this profile, after the scattering on impurities, happens due to emission of small-amplitude waves which go out slowly with the profile alignment, so that at the end of the simulations the profile becomes completely linear with a weakly modulated amplitude in the transverse direction [see Fig. 12(d)].

We define the transmission coefficient of a solitary wave $T$ as the ratio of its initial energy to the energy transmitted through the domain $\Lambda_{i}$, so that the quantity $T$ describes the solitary wave stability with respect to mass disorder. The dependence of $T$ on isotope mass $\mu_{i}$ is presented in Table IV. For comparison this table also contains the values for $T$ found from the simulations for the reduced 1D model governed by the Hamiltonian (11). As follows from Table IV, with the growth of the mass $\mu_{i}$, the transmission coefficient $T$ decreases. It is interesting to notice that the reduced 1D model gives for $\mu_{i} \leqslant 4$ approximately the same values of $T$ as the $2 \mathrm{D}$ model governed by the Hamiltonian (6). However, beginning from the value $\mu_{i}=5$, the $1 \mathrm{D}$ model gives the 

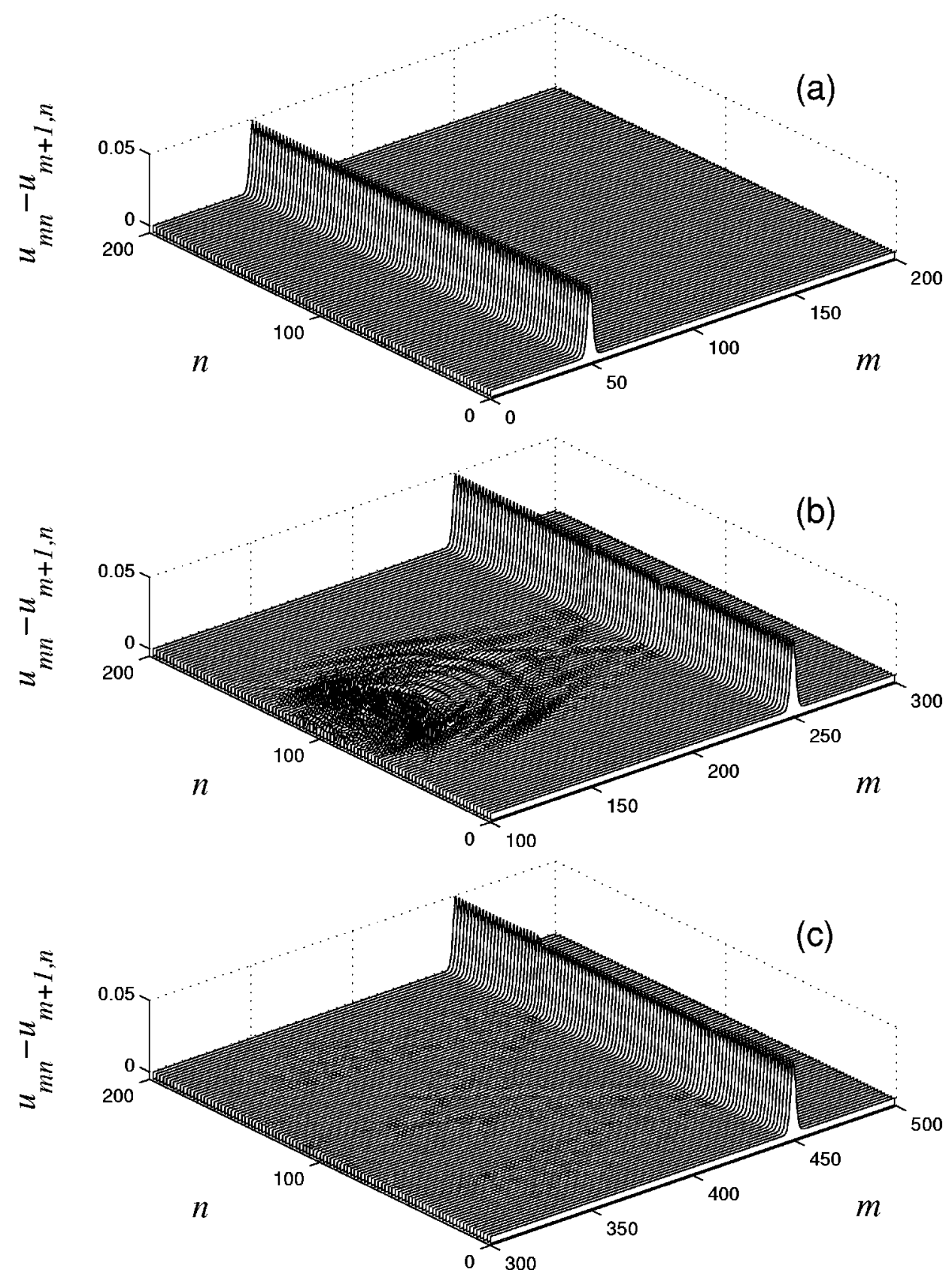

FIG. 10. Propagation of the solitary wave with velocity $s=1.5$ in the direction $\alpha=0^{\circ}$ through the vacancy situated at the $(100,100)$ th lattice site. Profiles of localized contractions $u_{m n}-u_{m+1, n}$ along the $X$ axis are presented (a) initially (at $\tau=0$ ), (b) at $\tau=66.68$, and (c) $\tau$ $=133.36$.

lesser values compared with the $2 \mathrm{D}$ case. This error is connected with the dimensionality of the reduced problem; in the $2 \mathrm{D}$ lattice the solitary wave has an opportunity to round isotope impurities of large mass and this is impossible in the 1D chain. This essential difference between the 2D lattice and the reduced $1 \mathrm{D}$ chain appears to be stronger when the isotope mass $\mu_{i}$ is larger. For instance, in the limit of infinitely large isotope mass $\mu_{i}$, in the reduced chain model the transmission coefficient $T$ is always zero (the soliton always reflects from a heavy impurity), while in the $2 \mathrm{D}$ lattice we always have $T>0$. In the latter case the solitary wave transmission occurs only because of streamlining immobile lattice sites.

In a finite lattice domain $\Lambda$ its boundary conditions should significantly affect the solitary wave dynamics. In order to examine the influence of transverse boundaries, we considered the solitary wave motion in the 2D lattice domain which was finite in the transverse $(n)$ direction (with free boundary conditions) but infinite in the longitudinal $(m)$ direction. To model the free boundary conditions, all the terms in the equations of motion which contain the bonds appearing outside the lattice domain $\Lambda$ were removed. Initially (at $\tau=0$ ), the solitary wave was taken with the velocity $s=1.5$. For $N=200$ the width of the solitary wave is $100 \sqrt{3}$ and its energy is $E=4.52$. The results of simulations of the solitary wave motion is presented in Fig. 12. The solitary wave motion is accompanied by intensive radiation of waves at the free boundaries. The edges of the solitary wave begin breaking and its profile acquires a nonlinear arched shape. The energy is translocated in the form of transverse waves from 

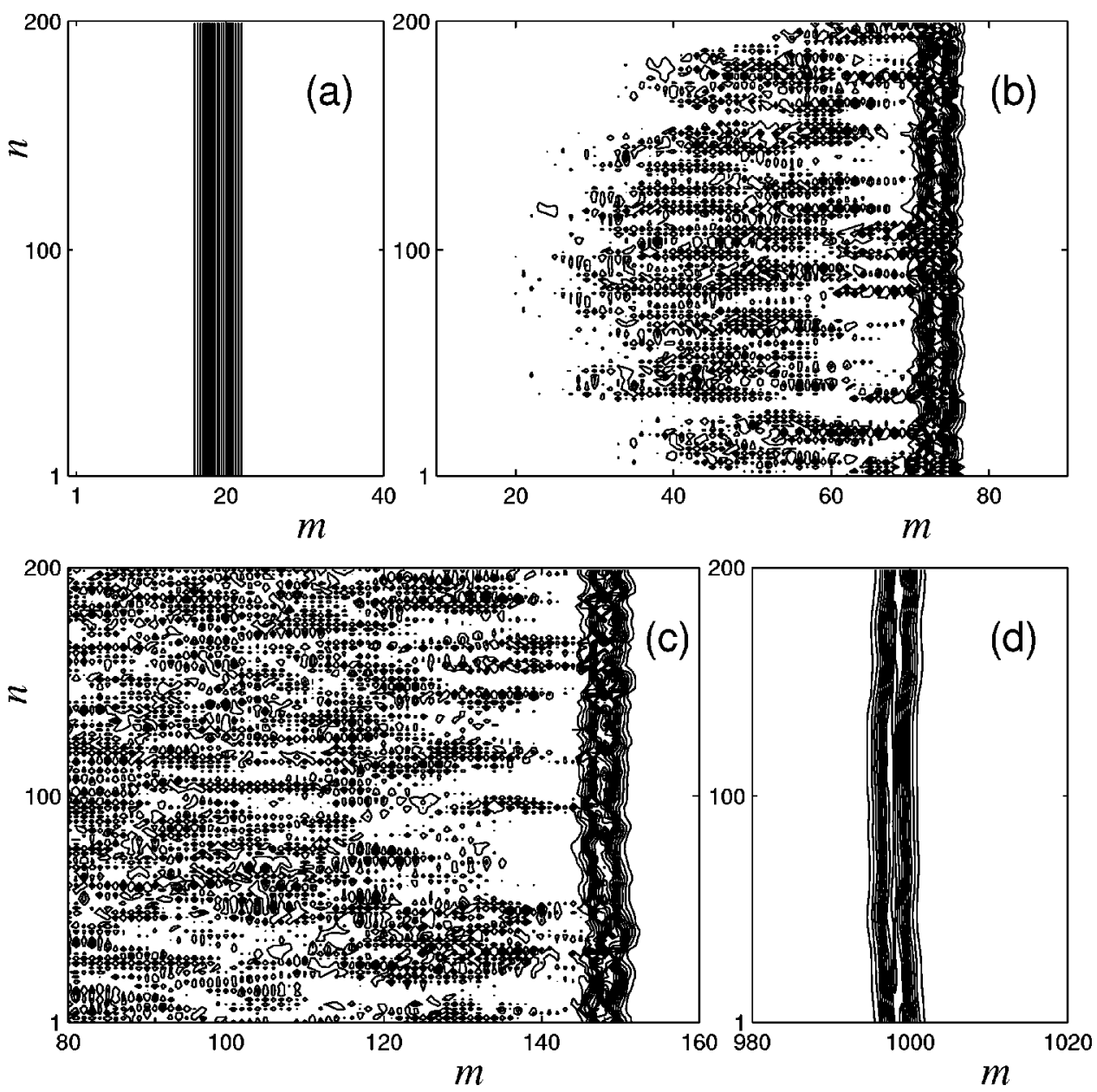

FIG. 11. Propagation of the solitary wave with velocity $s=1.5$ in the direction $\alpha=0^{\circ}$ through the lattice domain (40 $\leqslant m \leqslant 140$ ) with isotopic disorder (isotope mass is $\mu_{i}=3$ and the density of impurities is $c=1 / 50 \sqrt{3}$ ). Isolines of longitudinal localized contractions are presented (a) initially ( $\tau=0$ ), (b) at $\tau=18$, (c) $\tau=44$, and (d) $\tau=400$.

the solitary wave center to the edges and there they are emitted. The velocity, amplitude, and energy of the solitary wave monotonically diminish. Thus, at the time instants $\tau=338$, 1057, 1834, 3779 the energy was $E=3.73$ [Fig. 12(a)], 2.12 [Fig. 12(b)], 1.02, 0.09, respectively. Thus, in a finite 2D lattice the solitary wave has a finite lifetime which depends on the solitary wave width. In the example considered, the solitary wave of the width $100 \sqrt{3}$ losses the half energy during the time $\tau \simeq 1000$.

Let us consider now the motion of the solitary wave along the $Y$ axis $(\alpha=\pi / 2)$. To this end, we choose the following initial conditions:

$$
\begin{gathered}
u_{m n}=0, \quad v_{m n}(0)=\sum_{l=1}^{n} r\left[a\left(l-n_{0}\right)\right], \\
\dot{u}_{m n}=0, \quad \dot{v}_{m n}(0)=-s \sum_{l=1}^{n} r^{\prime}\left[a\left(l-n_{0}\right)\right],
\end{gathered}
$$

where the function $r(z)$ with $s>s_{0}$ is the soliton solution of the reduced system of Eqs. (15) and $n_{0}$ is the number of the site (along the $Y$ axis) at which the center of the solitary wave profile is situated. The period of the reduced chain is $a=\sqrt{3} / 2$.
The rectangular lattice domain $\Lambda$ was chosen with $M$ $=400$ and $N=200$. Similarly, for simulations of the equations of motion governed by the Hamiltonian (6) we use the periodic boundary conditions in the transverse $(X)$ direction and the fixed boundary conditions in the longitudinal $(Y)$ direction.

We choose again the velocity to be $s=1.5$ and consider the solitary wave propagation through a single vacancy situated at the $\left(m_{i}, n_{i}\right)$ th site with $m_{i}=200$ and $n_{i}=100$. The numerical integration of the equations of motion has shown that the propagation through the vacancy does not result immediately in decaying the solitary wave. At the vacancy, the solitary wave emits small-amplitude waves and a local deformation of its profile appears as shown in Fig. 13(a). Two modulational transverse waves appear that propagate along the solitary wave profile with emission of longitudinal smallamplitude waves [see Fig. 13(b)]. In the infinite lattice these modulational waves would move to infinity but in the transversely finite lattice domain $\Lambda_{i}$, due to the periodic boundary conditions, these waves collide many times and, as a result, the solitary wave looses its stability as illustrated by Fig. 13(c). Finally, the solitary wave looses $71 \%$ of its energy. Thus, initially (at $\tau=0$ ), the energy of the solitary wave of the width 200 sites was $E=8.15$ and after its propagation through the vacancy over 2700 lattice sites its energy 

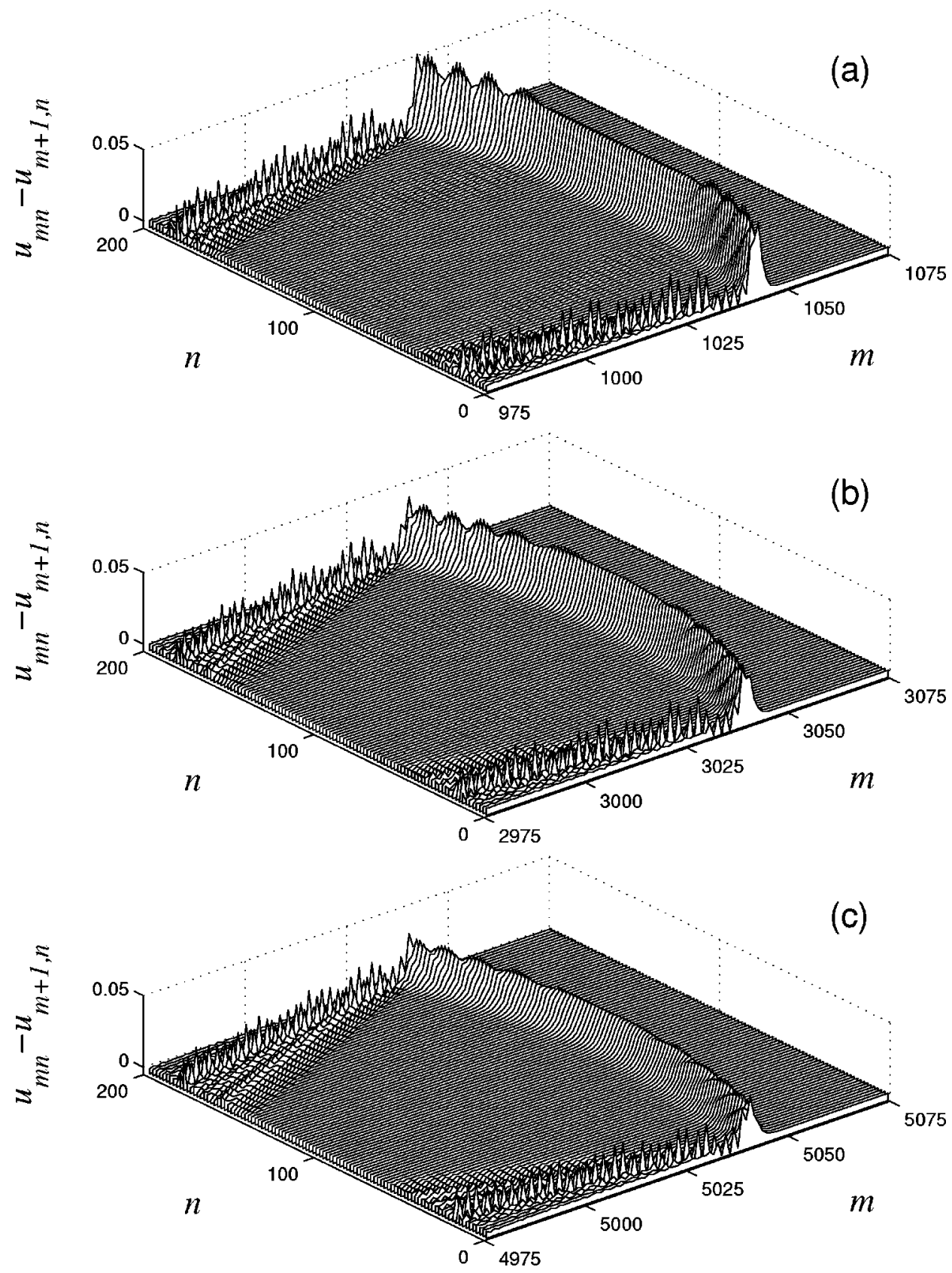

FIG. 12. Motion of the solitary wave with velocity $s=1.5$ in the direction $\alpha=0^{\circ}$ in transversely finite lattice domain with free boundary edges. Profiles of localized contractions are presented at time instants: (a) $\tau=338.46$, (b) $\tau=1057.08$, and (c) $\tau=1834.28$.

was $E=2.36$. Consequently, while moving along the direction with $\alpha=\pi / 2$, the solitary wave appears to be more sensitive to the presence of vacancies in the $2 \mathrm{D}$ lattice than in the direction with $\alpha=0$.

We consider now the solitary wave propagation in a lattice domain with isotopic disorder $\Lambda_{i}$. For simulations we chose $\Lambda_{i}$ to be the rectangular with $1 \leqslant m \leqslant 400$ and $40 \leqslant n$ $\leqslant 140$. For each integer $m$ we choose randomly the site number $n(m)$ from the interval $40 \leqslant n \leqslant 140$. We again set $\mu_{m, n(m)}=\mu_{i}$ where $\mu_{i}$ is an impurity mass and $\mu_{m n}=1$ for the other sites. Then in the domain $\Lambda_{i}$ the density of impurities is the same as before: $c=1 / 50 \sqrt{3}$. The solitary wave motion through the disordered domain $\Lambda_{i}$ is shown in Fig. 14. The dependence of the transmission coefficient $T$ on the impurity mass $\mu_{i}$ is presented in Table IV. The coefficient $T$ for solitary wave transmission in the direction with $\alpha=\pi / 2$ is always less than in the direction with $\alpha=0$. Contrary to the case with $\alpha=0$, the dependence of $T$ on $\mu_{i}$ for $\alpha=\pi / 2$ does not possess monotonic behavior. Thus, for $\mu_{i}=2$ the transmission coefficient is less than for $\mu_{i}=3$. Note that for the reduced 1D model the dependence of $T$ on $\mu_{i}$ is strictly monotonic. Therefore, the nonmonotonic behavior $T$ $=T\left(\mu_{i}\right)$ is a purely $2 \mathrm{D}$ effect: destroying a solitary wave occurs not only directly, while interacting with an impurity, but also under the interaction of transverse modulational waves appearing on the solitary wave profile.

Now we consider the solitary wave motion in the lattice domain $\Lambda$ which is infinite in the direction of the solitary wave propagation but finite in the transverse direction (with the free boundary conditions). We chose $M=400$ and $s$ 
TABLE IV. Dependence of the transmission coefficient $T$ for the solitary plane wave propagating through the lattice domain with isotopic disorder on dimensionless impurity mass $\mu_{i}$. The density of impurities is $c=1 / 50 \sqrt{3}$.

\begin{tabular}{|c|c|c|c|c|}
\hline \multirow[b]{3}{*}{$\mu_{i}$} & \multicolumn{4}{|c|}{$T$} \\
\hline & \multicolumn{2}{|l|}{$\alpha=0$} & \multicolumn{2}{|l|}{$\alpha=\pi / 2$} \\
\hline & Reduced 1D chain & 2D lattice & Reduced 1D chain & 2D lattice \\
\hline 1.0 & 1.0 & 1.0 & 1.0 & 1.0 \\
\hline 2.0 & 0.871 & 0.857 & 0.846 & 0.318 \\
\hline 3.0 & 0.697 & 0.681 & 0.656 & 0.407 \\
\hline 4.0 & 0.563 & 0.561 & 0.515 & 0.322 \\
\hline 5.0 & 0.470 & 0.489 & 0.414 & 0.259 \\
\hline 7.0 & 0.368 & 0.405 & 0.288 & 0.198 \\
\hline 10.0 & 0.279 & 0.348 & 0.188 & 0.163 \\
\hline$\infty$ & 0.0 & 0.254 & 0.0 & 0.104 \\
\hline
\end{tabular}

$=1.5$. The result of simulations of the solitary wave dynamics is presented in Fig. 15. Similarly to the case with $\alpha=0$, the propagation is accompanied by intensive emission of waves at the lattice edges. The solitary wave is not destroyed immediately but its velocity, amplitude, and energy monotonically decrease. Thus, at the time instants $\tau=0,176.6$, 371.4, 867.6, and 2106.8 the energy was $E=8.15,6.23$ [Fig. 15(a)], 3.18 [Fig. 15(b)], 0.88 [Fig. 15(c)], and 0.10, respectively. Therefore, for $\alpha=\pi / 2$ the lifetime of the solitary wave is significantly less than for $\alpha=0$. The solitary wave of the width 200 looses the half energy during the time $\tau$ $\simeq 300$.

\section{CONCLUSIONS}

Thus, the dynamics of solitary plane waves in a 2D hexagonal lattice has been investigated numerically by using pseudospectral techniques. Contrary to the previous studies, ${ }^{1}$ the lattice was considered to be purely isotropic. It is important that the solitary wave solutions we examined are quite narrow, even of one lattice spacing. Therefore we could study them at large supersonic velocities, say $s \simeq 1.5$, or even larger. We have found that the regime of the solitary wave propagation essentially depends on the direction of its motion; it is sensitive to microstructure of the lattice.

We have also carried out the extensive investigation of the solitary wave propagation through lattice domains with isotopic disorder. We have shown that the dynamical properties of the solitary wave significantly depend on the direction of its propagation. Thus, in the infinite 2D lattice the solitary wave is the most stable while moving along the bonds ( $\alpha=0, \pi / 3,2 \pi / 3, \pi, 4 \pi / 3,5 \pi / 3)$. When the lattice is finite in the transverse direction and the lattice edges are free, the lifetime of the solitary wave is the longest at these angles.

Contrary to the case of the 1D soliton theories on the soliton interaction with lattice impurities ${ }^{10-13}$ the important point of the 2D model considered in this paper is that within this model it is possible to model properly interactions of solitary waves with lattice vacancies. Here we have studied such types of interactions and found that the solitary waves of localized lattice deformation can easily round vacancies, while in a 1D chain such a situation does not make any sense.

\section{ACKNOWLEDGMENTS}

We thank J. C. Eilbeck for his constructive remarks and genuine interest in our work. This work was partially carried out with the financial support from the European Economic Community under the INTAS Grant No. 96-158. A.V.S. would like to express his thanks for the hospitality of the MIDIT Center and the Department of Mathematical Modelling of the Technical University of Denmark. Y.Z. wishes to acknowledge financial support from the Danish Research Academy.

\section{APPENDIX A: THE PSEUDOSPECTRAL METHOD}

Let us consider the following system of equations:

$$
\begin{aligned}
\ddot{r}_{n}= & \sum_{j}\left[U_{j}^{\prime}\left(\sum_{l=1}^{k_{j}} r_{n+l}\right)-U_{j}^{\prime}\left(\sum_{l=1}^{k_{j}} r_{n+l-1}\right)\right. \\
& \left.-U_{j}^{\prime}\left(\sum_{l=1}^{k_{j}} r_{n-k_{j}+l}\right)+U_{j}^{\prime}\left(\sum_{l=1}^{k_{j}} r_{n-k_{j}-1+l}\right)\right] .
\end{aligned}
$$

To find the whole class of soliton solutions as well as to calculate those values of velocity $s$ at which such solutions exist, we use the pseudospectral method developed by Eilbeck and Flesch $^{8}$ and Duncan et al. ${ }^{9}$ Since we look for traveling-wave solutions, $r_{n}(\tau)=r(z), z=n a-s \tau$, the set of Eqs. (A1) is reduced to

$$
\begin{aligned}
& s^{2} r^{\prime \prime}= \sum_{j=1}^{3}\left[U_{j}^{\prime}\left(\sum_{l=1}^{k_{j}} r(z+l a)\right)-U_{j}^{\prime}\left(\sum_{l=1}^{k_{j}} r[z+(l-1) a]\right)\right. \\
&-U_{j}^{\prime}\left(\sum_{l=1}^{k_{j}} r\left[z+\left(l-k_{j}\right) a\right]\right) \\
&\left.+U_{j}^{\prime}\left(\sum_{l=1}^{k_{j}} r\left[z+\left(l-k_{j}-1\right) a\right]\right)\right] \\
& n=0, \pm 1, \ldots
\end{aligned}
$$

As before, ${ }^{8,9}$ we look for solutions of Eq. (A2) in the form

$$
r(z) \simeq \sum_{p=0}^{N} c_{p} \phi_{p}(z)
$$

for some sufficiently large integer $N$ where the coefficients $\left\{c_{p}\right\}_{p=0}^{N}$ are to be determined. The basis functions $\phi_{p}(z)$ 's are chosen to be the same as in the cosine Fourier transform, i.e., $\phi_{p}(z)=\cos (2 \pi p z / L)$. We consider $r(z)$ to be a periodic function with a sufficiently large period $L$. In the limit of large $L$ we expect to obtain a good approximation to a solitary wave solution which has an infinite period. The boundary condition $r( \pm \infty)=0$ allows us to decrease the number of coefficients in the series (A3). Therefore we rewrite it in the form

$$
r(L / 2)=\sum_{p=0}^{N} c_{p} \cos (p \pi)=0
$$



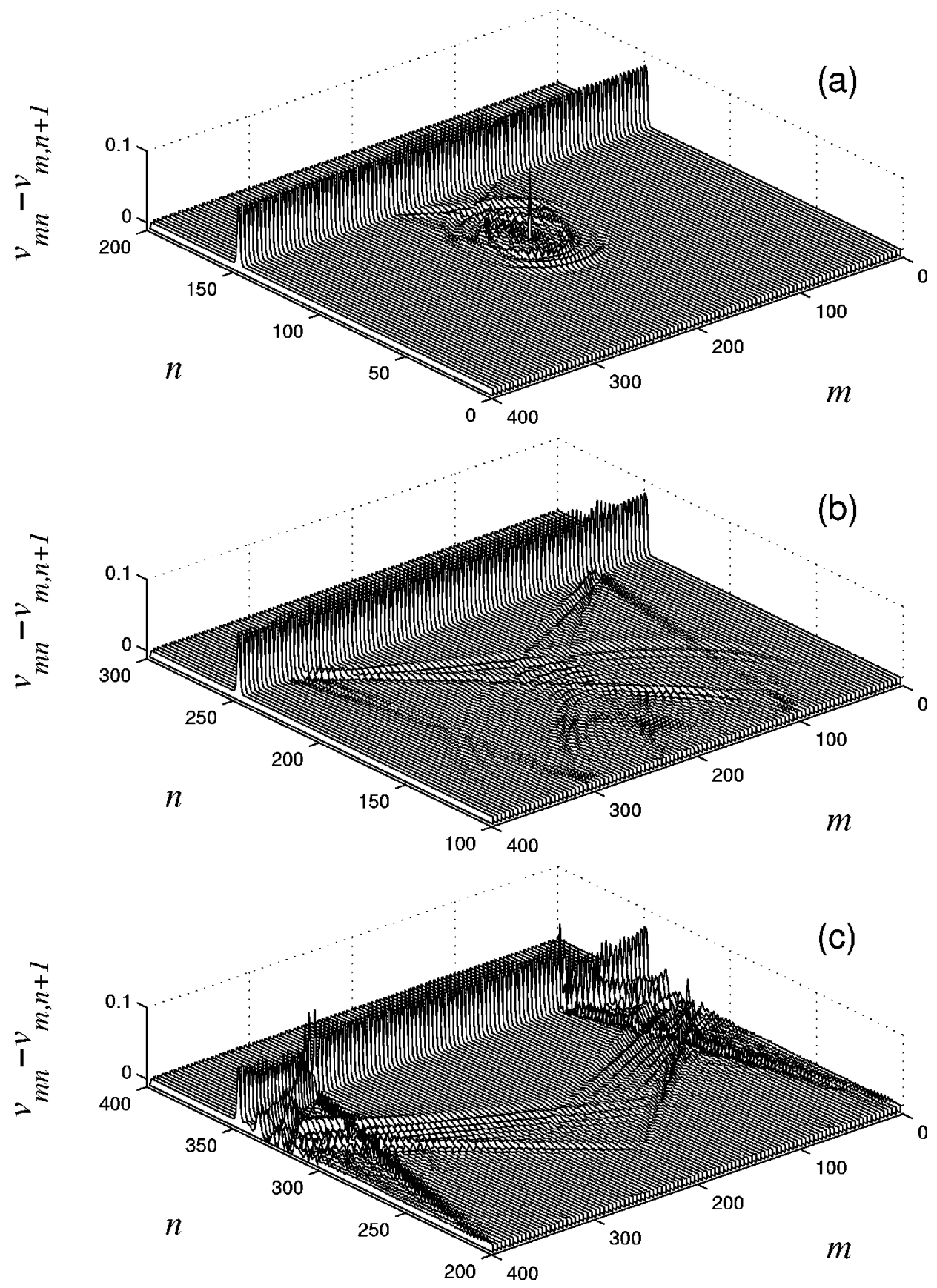

FIG. 13. Propagation of the solitary wave with velocity $s=1.5$ in the direction $\alpha=\pi / 2$ through the vacancy situated at the (200,100)th lattice site. Profiles of localized contractions $v_{m n}-v_{m+1, n}$ along the $Y$ axis are given at the times: (a) $(\tau=56.3),(\mathrm{b}) \tau=114.0$, and (c) $\tau$ $=137.8$.

and find the equality

$$
c_{0}=-\sum_{p=1}^{N} c_{p} \phi_{p}(L / 2) .
$$

Therefore Eq. (A3) can be rewritten as

$$
r(z) \simeq \sum_{p=1}^{N} c_{p}\left[\phi_{p}(z)-\phi_{p}(L / 2)\right]
$$

$$
\begin{aligned}
\mathcal{F}(z) \equiv & \sum_{p=1}^{N} c_{p} b_{p}(z)+\sum_{j=1}^{3}\left[U_{j}^{\prime}\left(\sum_{p=1}^{N} c_{p} q_{j p}^{(1)}(z)\right)\right. \\
& -U_{j}^{\prime}\left(\sum_{p=1}^{N} c_{p} q_{j p}^{(2)}(z)\right)-U_{j}^{\prime}\left(\sum_{p=1}^{N} c_{p} q_{j p}^{(3)}(z)\right) \\
& \left.+U_{j}^{\prime}\left(\sum_{p=1}^{N} c_{p} q_{j p}^{(4)}(z)\right)\right]=0
\end{aligned}
$$



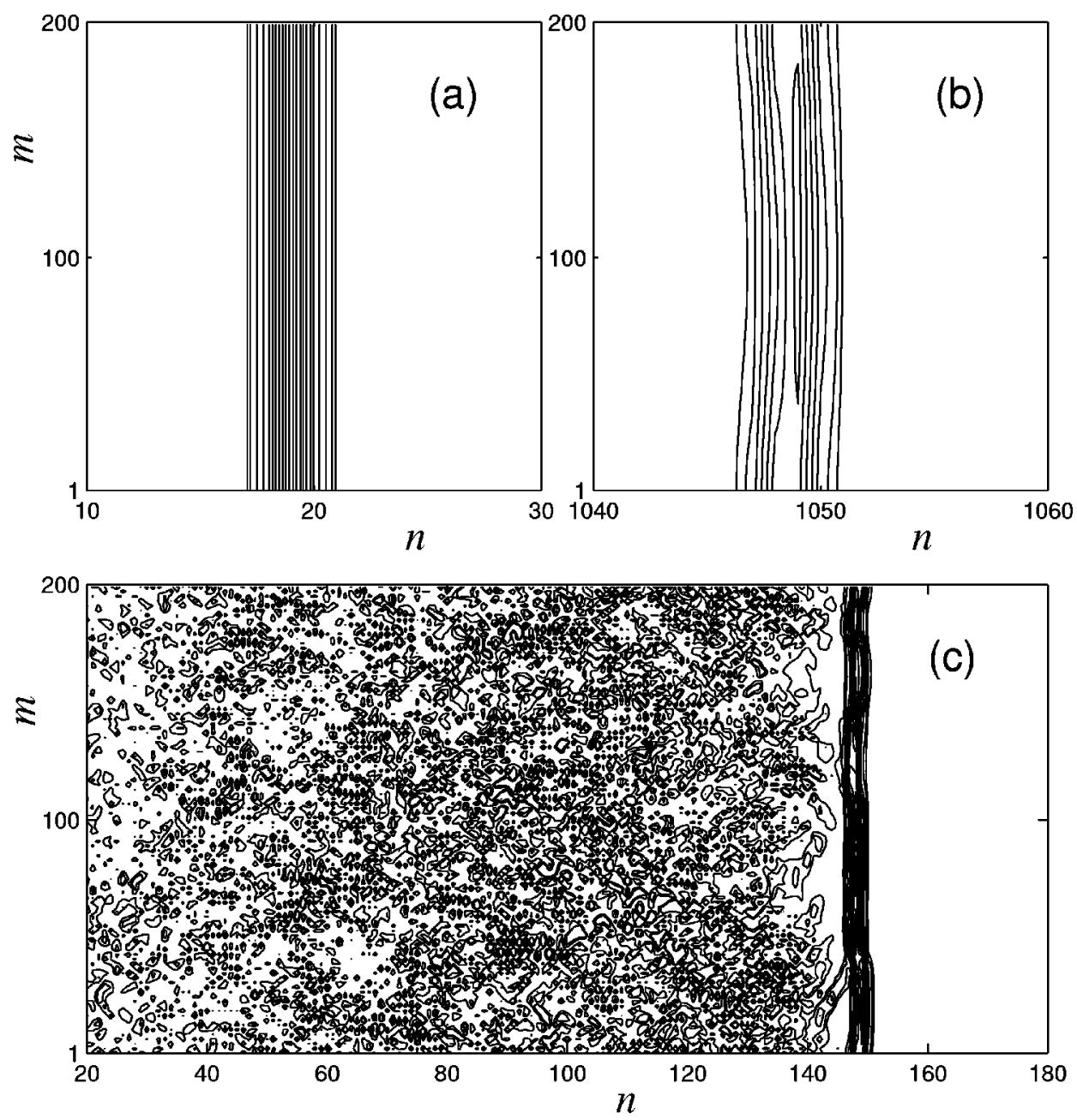

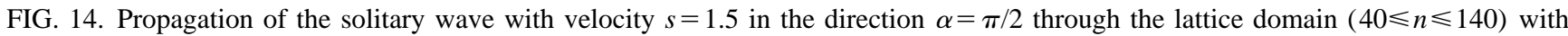
isotopic disorder (isotope mass is $\mu_{i}=3$ and density of impurities is $c=1 / 50 \sqrt{3}$ ). Isolines of longitudinal localized contractions are presented at time instants: (a) $(\tau=0)$, (b) at $\tau=670.89$, and (c) $\tau=78.39$.

$$
\begin{gathered}
b_{p}(z)=\left(\frac{2 \pi p s}{L}\right)^{2} \phi_{p}(z), \\
q_{j p}^{(1)}=\sum_{l=1}^{k_{j}}\left[\phi_{p}(z+l a)-\phi_{p}(L / 2)\right], \\
q_{j p}^{(2)}=\sum_{l=1}^{k_{j}}\left[\phi_{p}(z+(l-1) a)-\phi_{p}(L / 2)\right], \\
q_{j p}^{(3)}=\sum_{l=1}^{k_{j}}\left\{\phi_{p}\left[z+\left(l-k_{j}\right) a\right]-\phi_{p}(L / 2)\right\}, \\
q_{j p}^{(4)}=\sum_{l=1}^{k_{j}}\left\{\phi_{p}\left[z+\left(l-k_{j}-1\right) a\right]-\phi_{p}(L / 2)\right\} .
\end{gathered}
$$

Let us split the interval $[0, L / 2]$ into the $N$ collocation points:

$$
z_{i}=\frac{(i-1) L}{2 N}, \quad i=1,2, \ldots, N
$$

Then we obtain the system of $N$ nonlinear algebraic equations with respect to the $N$ coefficients $\left\{c_{p}\right\}_{p=1}^{N}$ :

$$
\mathcal{F}\left(z_{i}\right)=0, \quad i=1,2, \ldots, N
$$

This system of equations was solved numerically by the modification of the Powell hybrid method. The routine HYBRJ1 from the MINPAK package was used. We took the number of the Fourier coefficients $N=200$ and the period $L$ to be the solitary wave diameter multiplied by the factor of 10 . The important point in the numerical solution of Eq. (A10) was the choice of the initial guess. It was taken to be the function $-A \operatorname{sech}^{2}(\mu z)$ with the parameter values taken from the continuum approximation [see below Appendix B and expressions (B9) and (B10) there]. This approximation appears to be good enough for $s<1.2$. For higher velocities $s$ the parameters $A$ and $\mu$ were path followed from the interval $s<1.2$.

\section{APPENDIX B: THE SOLITARY WAVE PROFILE IN THE CONTINUUM APPROXIMATION}

Let us consider the system of the equations of motion (16). Suppose their solution $r_{n}(\tau)$ depends smoothly on the site number $n$. Next, we set $n a=x$, so that the profile $r_{n}(\tau)=r(x, \tau)$ changes smoothly with the change of the variable $x$. Then for any integer $l$ in the continuum approxima- 

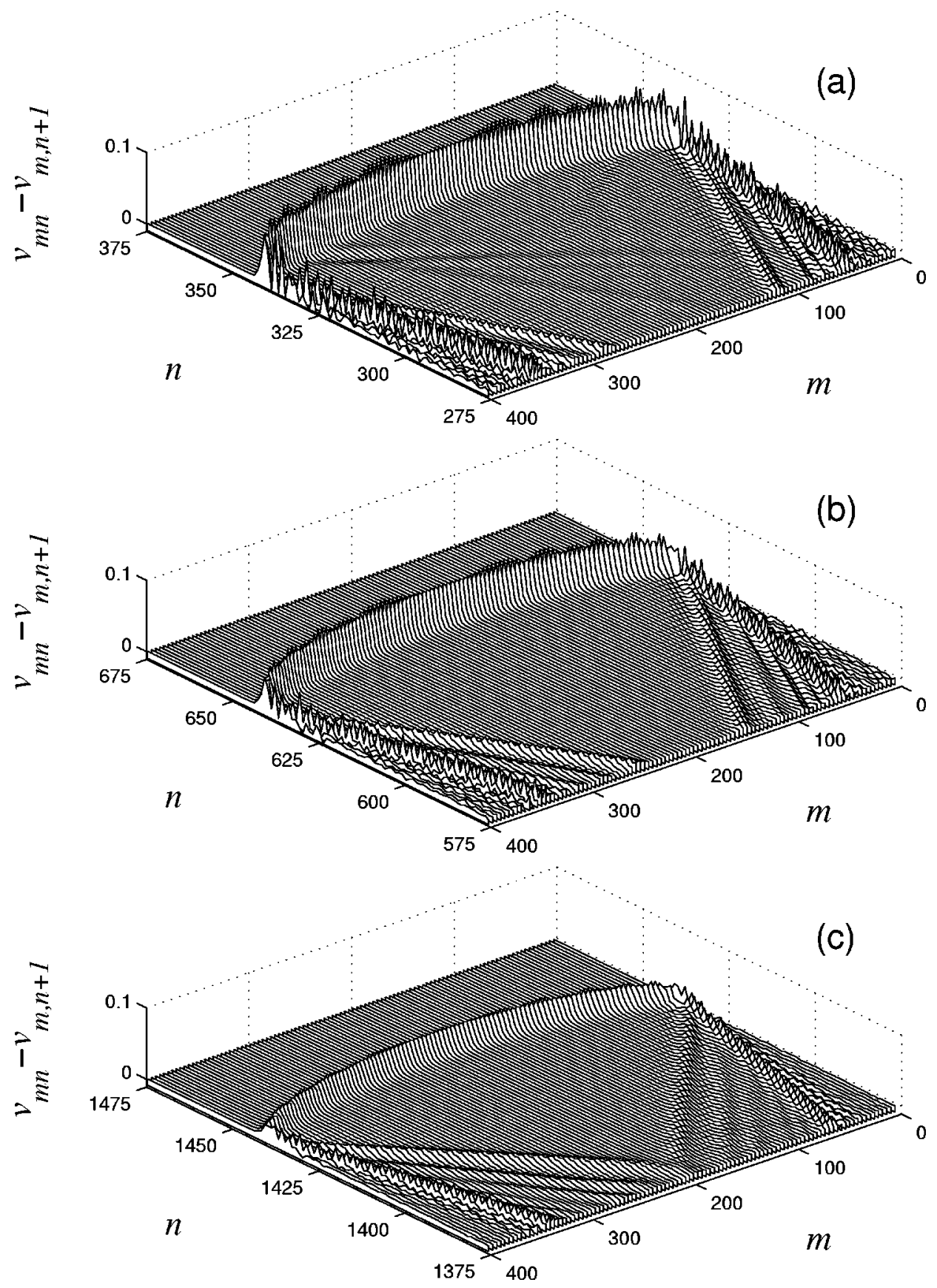

FIG. 15. Motion of the solitary wave with velocity $s=1.5$ in the direction $\alpha=\pi / 2$ in the transversely finite lattice domain with free boundary edges. Profiles of localized contractions are given at time instants: (a) $\tau=176.56$, (b) $\tau=371.36$, and (c) $\tau=867.58$.

tion we can write the following expansion:

$$
\begin{aligned}
r_{n \pm l}(\tau) \simeq & r \pm l a r_{x}+\frac{1}{2}(l a)^{2} r_{x x} \pm \frac{1}{6}(l a)^{3} r_{x x x}+\frac{1}{24}(l a)^{4} r_{x x x x} \\
& +\ldots
\end{aligned}
$$

Next, we substitute this expansion into Eqs. (16) and keep all the expansion terms up to fourth order. However, we linearize all the terms which contain fourth-order derivatives over $x$, omitting anharmonic fourth-order terms. As a result, we obtain the following partial differential equation:

$$
r_{\tau \tau}-\sum_{j}\left[d_{j} a U_{j}^{\prime}\left(k_{j} r\right)+\frac{1}{12} d_{j}^{6} r_{x x}\right]_{x x}=0, \quad d_{j}=k_{j} a
$$

Here the linear fourth-order term $r_{x x x x}$ takes in the harmonic approximation account of the lattice dispersion caused by its discreteness. Omitting this dispersive term for smallamplitude waves $r \ll a$ this equation is reduced to the linear wave equation

$$
r_{\tau \tau}-\sum_{j} d_{j}^{4} r_{x x}=0
$$


from which one can find the velocity of small-amplitude plane waves propagating in the direction given by the angle $\alpha$ :

$$
s_{0}=\sqrt{d_{1}^{4}+d_{2}^{4}+d_{3}^{4}}=a^{2} \sqrt{k_{1}^{4}+k_{2}^{4}+k_{3}^{4}} .
$$

Taking Eqs. (9) into account, we can calculate this value explicitly. As a result, we find

$$
s_{0}=\sqrt{9 / 8}=1.06066 \ldots,
$$

so that the characteristic (sound) velocity, i.e., the velocity of plane long-wave waves in the $2 \mathrm{D}$ hexagonal isotropic lattice, does not depend on the direction of their propagation.

Suppose now that the function $r(x, \tau)$ is a solitary wave solution, i.e., $r(x, \tau)=r(z)$ with $z=x-s \tau$, and $r, r^{\prime}$, and $r^{\prime \prime} \rightarrow 0$ when $z \rightarrow \infty$. Then, Eq. (B2) can be twice integrated and transformed to the following generalized Boussinesq equation:

$$
\left(a^{4} / 12\right) \sum_{j} k_{j}^{6} r^{\prime \prime}+\sum_{j} k_{j} U_{j}^{\prime}\left(k_{j} r\right)-(s / a)^{2} r=0 .
$$

This equation describes the dynamics of chain particles, each of which interacts with six neighbors located at the distances $d_{j}=k_{j} a, j=1,2,3$. Expanding the reduced LJ potential (12) up to the third order (taking into account only cubic anharmonicity):

$$
U_{j}(r)=\frac{1}{2} d_{j}^{2} r^{2}-d_{j}\left(4 d_{j}^{2}-\frac{1}{2}\right) r^{3}+\cdots,
$$

and using this expansion in Eq. (B6), we obtain the standard Boussinesq equation

$$
\frac{1}{12} \sum_{j} d_{j}^{6} r^{\prime \prime}+\left(s_{0}^{2}-s^{2}\right) r-\frac{3}{a} \sum_{j} d_{j}^{4}\left(4 d_{j}^{2}-\frac{1}{2}\right) r^{2}=0
$$

which has a soliton solution of the form

$$
r(z)=-A \operatorname{sech}^{2}(\mu z)
$$

where

$$
\begin{gathered}
\mu^{2}=3\left(s^{2}-s_{0}^{2}\right) / \sum_{j} d_{j}^{6}, \\
A=\left(s^{2}-s_{0}^{2}\right) a / \sum_{j} d_{j}^{4}\left(8 d_{j}^{2}-1\right), \\
s>s_{0}, \quad d_{j}=k_{j} a .
\end{gathered}
$$

The distances $d_{j}$ and integers $k_{j}, j=1,2$, and 3 , are given by Eqs. (9) and (10).

The necessary condition for the usage of the continuum approximation is the requirement of a sufficient "thickness", (diameter $D$ ) of the soliton solution. In fact, this approximation can be used only for $1<s / s_{0}<1.05$. For higher velocities the discreteness of the chain should be taken into account more properly. For this purpose we use the pseudospectral method already described in Appendix A.
${ }^{1}$ D. B. Duncan, J. C. Eilbeck, C. H. Walshaw, and V. E. Zakharov, Phys. Lett. A 158, 107 (1991).

${ }^{2}$ J. A. D. Wattis, Phys. Scr. 50, 238 (1994).

${ }^{3}$ O. A. Druzhinin and L. A. Ostrovsky, Phys. Lett. A 160, 357 (1991).

${ }^{4}$ L. A. Ostrovsky, L. A. Papko, and V. V. Stepanyants, Sov. Phys. JETP 51, 417 (1980).

${ }^{5}$ J. Pouget, Phys. Rev. B 43, 3575 (1991); 43, 3582 (1991).

${ }^{6}$ J. Pouget, Phys. Rev. B 46, 10554 (1992).

${ }^{7}$ R. Huss, F. G. Mertens, and Yu. B. Gaididei, in Fluctuation Phenomena: Disorder and Nonlinearity, edited by A. R. Bishop, S.
Jiménez, and L. Vázquez (World Scientific, Singapore, 1995), p. 244.

${ }^{8}$ J. C. Eilbeck and R. Flesch, Phys. Lett. A 149, 200 (1990).

${ }^{9}$ D. B. Duncan, J. C. Eilbeck, H. Feddersen, and J. A. D. Wattis, Physica D 68, 1 (1993).

${ }^{10}$ Q. Li, St. Pnevmatikos, E. N. Economou, and C. M. Soukoulis, Phys. Rev. B 37, 3534 (1988).

${ }^{11}$ Q. Li, C. M. Soukoulis, St. Pnevmatikos, and E. N. Economou, Phys. Rev. B 38, 11888 (1988).

${ }^{12}$ Yu. S. Kivshar, Phys. Rev. A 43, 3117 (1991).

${ }^{13}$ Z. Fei, Yu. S. Kivshar, and L. Vázquez, Phys. Rev. A 45, 6019 (1992). 